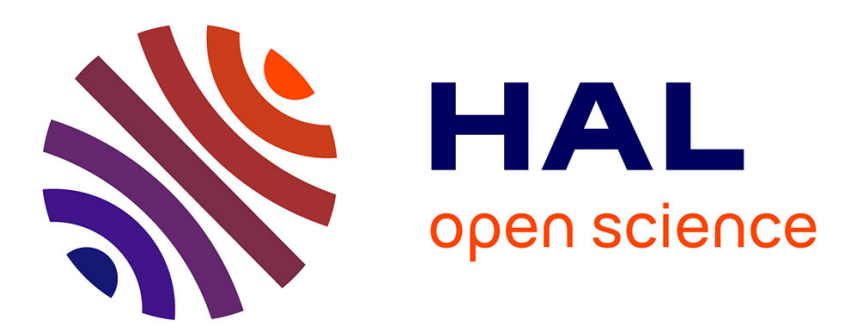

\title{
Central Frequency Tuning Considerations for Gm-C Resonators in GaAs Technology
}

Emilie Avignon, Sylvie Guessab, Richard Kielbasa

\section{To cite this version:}

Emilie Avignon, Sylvie Guessab, Richard Kielbasa. Central Frequency Tuning Considerations for GmC Resonators in GaAs Technology. IEEE-MWSCAS Midwest Symposium on Circuits and Systems, Aug 2008, Knoxville, United States. pp.558-561, 10.1109/MWSCAS.2008.4616860 . hal-00280535

\section{HAL Id: hal-00280535 \\ https://hal-centralesupelec.archives-ouvertes.fr/hal-00280535}

Submitted on 19 May 2008

HAL is a multi-disciplinary open access archive for the deposit and dissemination of scientific research documents, whether they are published or not. The documents may come from teaching and research institutions in France or abroad, or from public or private research centers.
L'archive ouverte pluridisciplinaire HAL, est destinée au dépôt et à la diffusion de documents scientifiques de niveau recherche, publiés ou non, émanant des établissements d'enseignement et de recherche français ou étrangers, des laboratoires publics ou privés. 


\title{
Central Frequency Tuning Considerations for Gm-C Resonators in GaAs Technology
}

\author{
Emilie Avignon, Sylvie Guessab, Richard Kielbasa \\ Supélec, Department of Signals and Electronic Systems \\ 3, rue Joliot Curie \\ 91192 Gif-sur-Yvette Cedex \\ Email: Emilie.Avignon@supelec.fr, Sylvie.Guessab@supelec.fr, Richard.Kielbasa@supelec.fr
}

\begin{abstract}
The ability of adjustment of the central frequency of a $\mathrm{Gm}-\mathrm{C}$ resonator in GaAs technology is discussed. First, it is shown that whatever the technology the wanted quality factor can be reached through an appropriate sizing of the transconductance values, with adding an external feedback capacitor in the transconductance amplifiers. Then, it is demonstrated that the adjustment of the central frequency must be made preferentially by a specifical transconductance. Finally, because in GaAs technologies varying the transconductance values leads to current offsets which could damage the performances, the maximal allowed current offsets are determined. To illustrate these considerations, simulation at transistor level of a proposed structure in GaAs P-HEMT 0.2 $\mu \mathrm{m}$ with integrated feedback capacitors is presented and demonstrates a tunable central frequency from 750 to $810 \mathrm{MHz}$.
\end{abstract}

\section{INTRODUCTION}

The Gm-C resonator, based on a gyrator structure, is a solution currently investigated because of its reduced integration cost and its possibly high quality factor [1]-[3]. In GaAs, $\mathrm{Gm}-\mathrm{C}$ resonator structures are different from conventional CMOS technology, due to the absence of P-type transistors. Their realizations require either current mirrors, difficult to implement [5], or static current sources. Tuning the central frequency is generally performed through changes in the bias current of the transconductance amplifiers. When the structure contains current mirror, this adjustment is done without additional devices. When the structure contains simple current sources, servo-control of the bias point [6], expensive in terms of complexity and consumption, is necessary. Otherwise, if no further device is wished, one may choose to tune the central frequency considering the tolerance to current offsets.

This is one of the points discussed in this paper, where the maximal current offset tolerance is analytically determined. The ability of tuning the central frequency with tolerance to current offset is also evaluated through transistor level simulations. Besides, using the expression of the quality factor shown in [4], it is proposed in this paper to add external feedback capacitors on transconductance amplifiers of Gm-C resonator structure in order to obtain the desired quality factor. This principle allows the designer to use weak load resistances instead of high impedance current source, simplifying the realization.

In the first part of the paper, the structure of a classical $\mathrm{Gm}-\mathrm{C}$ resonator in GaAs technology is shown. A small- signal model and the transfer function are given. Design methodological elements to reach the desired quality factor are presented, which are valid whatever the technology. The second part focuses on the tuning of the central frequency. Several means are listed and discussed. It is shown that in most cases, it is preferable to tune the central frequency by varying one of the transconductances, which is generally made by varying the bias current of the transconductance amplifier. The maximal allowed current offsets are evaluated. In the last part, the previous principles are illustrated through a circuit using the maximal allowed current offsets for the adjustment of the central frequency from $750 \mathrm{MHz}$ to $810 \mathrm{MHz}$.

\section{SMALL-SIGNAL MODEL AND DESIGN METHODOLOGICAL ELEMENTS}

The general structure of a $\mathrm{Gm}-\mathrm{C}$ resonator in $\mathrm{GaAs}$ technology is presented in fig. 1. It is composed of two transconductance amplifiers $\left(G_{m 1}\right.$ et $\left.G_{m 2}\right)$ and two integrated capacitors $C$. The bias current sources $\left(I_{01}\right.$ and $\left.I_{02}\right)$ must be equal on the upper part and on the lower part of the circuit to get only a dynamic current at the output of $G_{m 1}$ and $G_{m 2}\left(i_{1}, i_{2}\right)$. Then, level shifters restore the suitable bias voltage before entering the next stage.

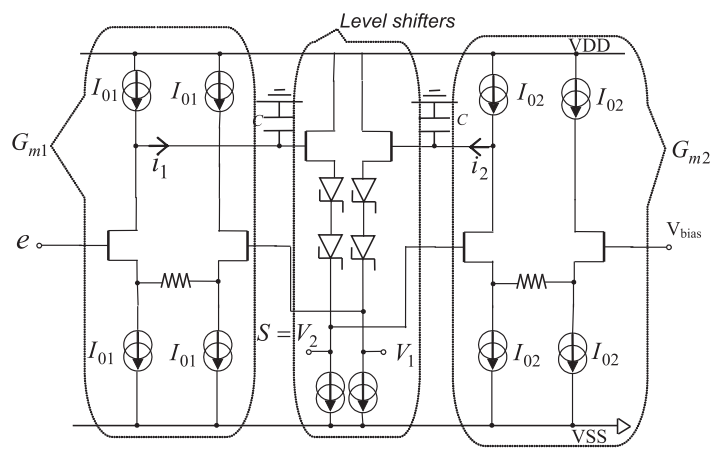

Fig. 1. General structure of Gm-C resonator in GaAs technology

The small-signal model, simplified but enough for a good comprehension of the circuit, is depicted in fig. 2. The level shifter stages are modelised by the attenuations $A 1$ and $A 2$. Other parameters which appear in this model are the transconductance values $G_{m 1}$ and $G_{m 2}$, the integrated capacitors $C$, the output parasitic capacitors of the transconductance 


$$
\frac{V_{2}(s)}{E(s)}=\frac{A_{1}\left(s^{2} C_{f 1}\left(C_{f 2}+C_{2}\right)+s\left(-G m_{1}\left(C_{f 1}+C_{2}\right)+\frac{C_{f 1}}{R_{2}}\right)-\frac{G m_{1}}{R_{2}}\right)}{s^{2}\left(C_{f 1}+C_{1}\right)\left(C_{f 2}+C_{2}\right)+s\left[\frac{1}{R_{1}}\left(C_{2}+C_{f 2}\right)+\frac{1}{R_{2}}\left(C_{1}+C_{f 1}\right)-C_{f 2} G m_{1} A_{1} A_{2}\right]+\frac{1}{R_{1} R_{2}}+G m_{1} G m_{2} A_{1} A_{2}}
$$

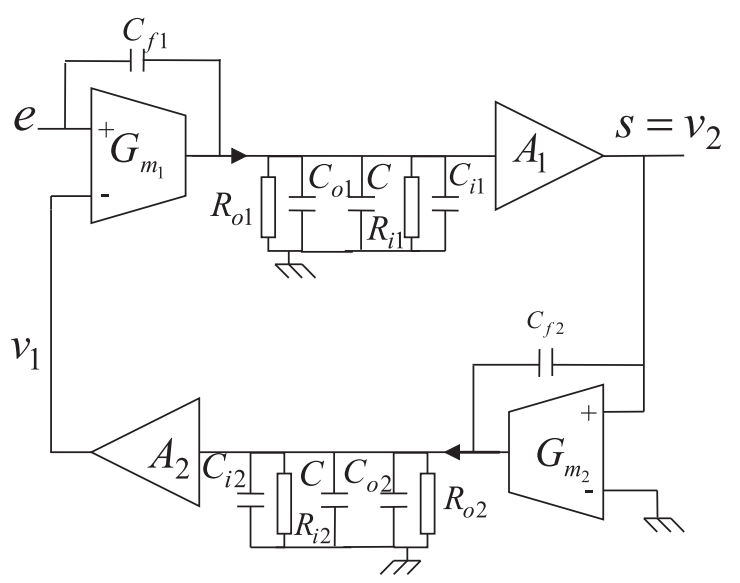

Fig. 2. Small-signal model of the resonator

amplifier stages $\left(C_{o 1}, C_{o 2}\right)$, the input parasitic capacitors of the level shifters stages $\left(C_{i 1}, C_{i 2}\right)$, the output resistances of the transconductance amplifier stages $\left(R_{o 1}\right.$ and $\left.R_{o 2}\right)$, the input resistances of level shifter stages $\left(R_{i 1}, R_{i 2}\right)$ and the feedback parasitic capacitors of the transconductance amplifiers $\left(C_{f 1}\right.$ and $C_{f 2}$ ). The transfer function of the resonator can be calculated from this model. It is given by the formula (1).

where:

$$
\left\{\begin{array}{l}
C_{1}=C+C_{o 1}+C_{i 1} \\
C_{2}=C+C_{o 2}+C_{i 2}
\end{array}\right.
$$

and :

$$
\left\{\begin{array}{l}
R_{1}=R_{o 1} / / R_{i 1} \\
R_{2}=R_{o 2} / / R_{i 2}
\end{array}\right.
$$

This transfer function presents three terms : one high-pass term, one band-pass term and one low-pass term. Only the band-pass term is useful for the resonator. If necessary, the other terms could be corrected by simple first-order high-pass and low-pass filters. The central pulsation $\left(\omega_{0}\right)$ and the quality factor $(Q)$, deduced from (1) are given by (2) and (3):

$$
\omega_{0}=\sqrt{\frac{1+G_{m 1} G_{m 2} A_{1} A_{2} R_{1} R_{2}}{R_{1} R_{2}\left(C_{1}+C_{f 1}\right)\left(C_{2}+C_{f 2}\right)}}
$$

$Q=\frac{\sqrt{R_{1} R_{2}\left(C_{1}+C_{f 1}\right)\left(C_{2}+C_{f 2}\right)\left(1+G_{m 1} G_{m 2} A_{1} A_{2} R_{1} R_{2}\right)}}{R_{2}\left(C_{2}+C_{f 2}\right)+R_{1}\left(C_{1}+C_{f 1}\right)-C_{f 2} G_{m 1} A_{1} A_{2} R_{1} R_{2}}$

Usually, $G_{m 1}=G_{m 2}=G_{m}, C_{1}=C_{2}=C$ for design considerations and $C_{f 2}$ and $C_{f 2}$ are negligible in front of $C_{1}$ and $C_{2}$. In CMOS common structure, $A_{1}=A_{2}=1$, so generally the central frequency has to be sized by $C$ and $G_{m}$ according to equation (2). From the expression (3), it may be noticed that $Q$ is even more important that the output resistances of the transconductance amplifiers stage are high. This determines the choice of some structures as cascod amplifiers, current sources or current mirrors with high output resistances. But, another effect can increase the quality factor: the parasitic feedback capacitor $C_{f 2}$ introduces a negative term in the denominator, depending on $G_{m 1}$, which can bring up the quality factor to very high values (to infinity or even cause instability if the denominator becomes negative). This is a very interesting effect for designing $\mathrm{Gm}-\mathrm{C}$ resonator with a desired quality factor. With adding an external feedback it is possible, for a given central frequency, associated to a value of product $G_{m 1} G_{m 2}$, to determine the respective values of $G_{m 1}$ and $G_{m 2}$ to reach the desired quality factor. For example in fig. 3, the gain response obtained for three combinations of $G_{m 1}$ and $G_{m 2}$ is plotted with the following parameters : $R_{o 1}=R_{o 2}=1$ $k \Omega, R_{i 1}=R_{i 2}=10 \mathrm{k} \Omega, C_{o 1}=C_{o 2}=0.2 \mathrm{pF}, C_{i 1}=C_{i 2}=0.1$ $\mathrm{pF}, C=1.2 \mathrm{pF}$ and $C_{f 1}=C_{f 2}=0.3 \mathrm{pF}$. These plots show that,

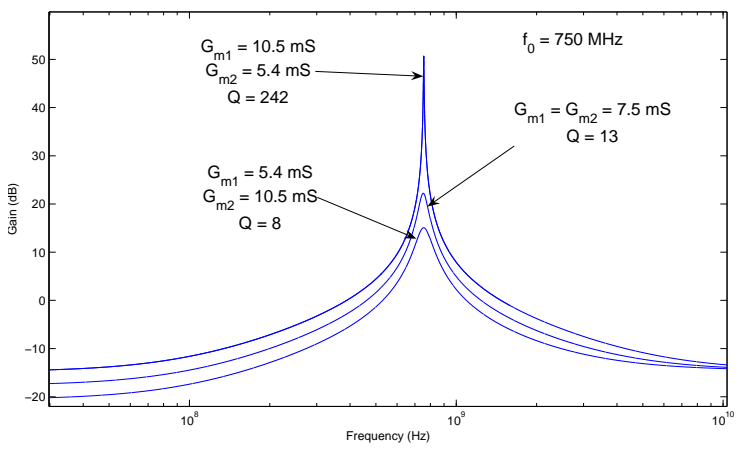

Fig. 3. Gain response varying $G_{m 1}$ at constant $f_{0}\left(G_{m 1} G_{m 2}\right.$ constant)

for a fixed value of $\left(C_{f 2}\right)$, the quality factor can be enhanced by increasing $G_{m 1}$ and keeping product $G_{m 2} G_{m 1}$ constant. As well, with given $G_{m 1}$ and $G_{m 2}$, we can determine $C_{f 2}$, keeping the sum $\left(C_{2}+C_{f 2}\right)$ constant, to obtain the desired $Q$. This observations leads us to focus on structures on which we add external feedback capacitor to get better quality factor. Of course this capacitor has an influence on the central frequency and should be taken into account right from the sizing.

\section{DISCUSSION ON THE ABILITY OF TUNING}

\section{A. Choice of tuning}

It clearly appears from equation (2) and this is a classical result, that the central frequency can be controled through the adjustment of the transconductances values $\left(G_{m 1}\right.$ and/or $G_{m 2}$ ). This can be done by varying $I_{01}$ and $I_{02}$. It is also possible to tune the central frequency through varactors 


$$
\begin{aligned}
& S(s)=\frac{A_{1} s^{2} C_{f 1}\left(C_{f 2}+C_{2}\right)+s-G m_{1}\left(C_{f 1}+C_{2}\right)+\frac{C_{f 1}}{R_{2}}-\frac{G m_{1}}{R_{2}}}{s^{2}\left(C_{f 1}+C_{1}\right)\left(C_{f 2}+C_{2}\right)+s\left[\frac{1}{R_{1}}\left(C_{2}+C_{f 2}\right)+\frac{1}{R_{2}}\left(C_{1}+C_{f 1}\right)-C_{f 2} G m_{1} A_{1} A_{2}\right]+\frac{1}{R_{1} R_{2}}+G m_{1} G m_{2} A_{1} A_{2}} E(s) \\
& -\frac{A_{1}\left(s\left(C_{f 2}+C_{2}\right)+1 / R_{2}\right)}{s^{2}\left(C_{f 1}+C_{1}\right)\left(C_{f 2}+C_{2}\right)+s\left[\frac{1}{R_{1}}\left(C_{2}+C_{f 2}\right)+\frac{1}{R_{2}}\left(C_{1}+C_{f 1}\right)-C_{f 2} G m_{1} A_{1} A_{2}\right]+\frac{1}{R_{1} R_{2}}+G m_{1} G m_{2} A_{1} A_{2}} \Delta I_{01}(s) \\
& -\frac{A_{1} A_{2} G_{m 1}}{s^{2}\left(C_{f 1}+C_{1}\right)\left(C_{f 2}+C_{2}\right)+s\left[\frac{1}{R_{1}}\left(C_{2}+C_{f 2}\right)+\frac{1}{R_{2}}\left(C_{1}+C_{f 1}\right)-C_{f 2} G m_{1} A_{1} A_{2}\right]+\frac{1}{R_{1} R_{2}}+G m_{1} G m_{2} A_{1} A_{2}} \Delta I_{02}(s)
\end{aligned}
$$

$\left(C_{1}, C_{f 1}, C_{2}, C_{f 2}\right)$, but it is not commonly used because varactors have poor capacity variation range. A less classical result, typical of GaAs structures, is that the central frequency may also be tuned by the level shifters attenuation, but this ability will not be discussed here. If varying $G_{m 1}$ or $G_{m 2}$ will vary the central frequency, it will also change the quality factor. To determine which of $G_{m 1}$ or $G_{m 2}$ has the stronger influence on $Q$, the quality factor is differentiated over $G_{m 1}$ and $G_{m 2}$.

$$
\begin{gathered}
\frac{\partial Q}{\partial G_{m 1}}=\frac{a b G_{m 2}}{\sqrt{a\left(1+G_{m 1} G_{m 2} b\right)}\left(c-b C_{f 2} G_{m 1}\right)} \\
+\frac{\sqrt{a\left(1+b G_{m 1} G_{m 2}\right)} b C_{f 2}}{\left(c-b C_{f 2} G_{m 1}\right)^{2}} \\
\frac{\partial Q}{\partial G_{m 2}}=1 / 2 \frac{a b G_{m 1}}{\sqrt{a\left(1+b G_{m 2} G_{m 1}\right)}\left(c-b C_{f 2} G_{m 1}\right)}
\end{gathered}
$$

where:

$$
\left\{\begin{array}{l}
a=R_{1} R_{2}\left(C_{1}+C_{f 1}\right)\left(C_{2}+C_{f 2}\right) \\
b=A_{1} A_{2} R_{1} R_{2} \\
c=R_{2}\left(C_{2}+C_{f 2}\right)+R_{1}\left(C_{1}+C_{f 1}\right)
\end{array}\right.
$$

In order to know which way of tuning leads to have a more constant quality factor, the sign of $K=\frac{\partial Q}{\partial G_{m 1}}-\frac{\partial Q}{\partial G_{m 2}}$ must be considered. If $G_{m 2} \geq G_{m 1}$, it is better to tune $G_{m 2}$ because $K$ is positive whatever $a, b$ and $c$, which means that variations of $G_{m 1}$ have more impact on $Q$ than variations on $G_{m 2}$. Otherwise, when $G_{m 2}<G_{m 1}$ it could be better to tune $G_{m 1}$.

\section{B. Maximal allowed offsets}

From a small-signal model point of view, it has been seen that it is possible to adjust the value of the central frequency by the value of transconductances $G_{m 2}$ or $G_{m 1}$. For general structure in GaAs (fig. 1), when the value of the bias current source are changed $\left(I_{02}+\Delta I_{02}\right.$ or $\left.I_{01}+\Delta I_{01}\right)$, a current offset will appear $\left(\Delta I_{01}\right.$ or $\left.\Delta I_{02}\right)$. These offsets can lead to degradations because the functioning ranges of transconductance amplifiers are limited. Here, the maximal current offsets for a good fonctionning of the transconductance amplifiers will be determined. For that, the offsets can be modelled as perturbations like presented in fig. 4. The total expression of the output of the resonator, including the perturbations can be expressed by the formula (6). The denominators of the transfer functions towards the perturbations are the same that toward the signal, which indicates that they will not lead to instability if the resonator was previously stable. The impact of the two current offsets on the output voltage of the resonator $\left(\Delta V_{2}\right)$,

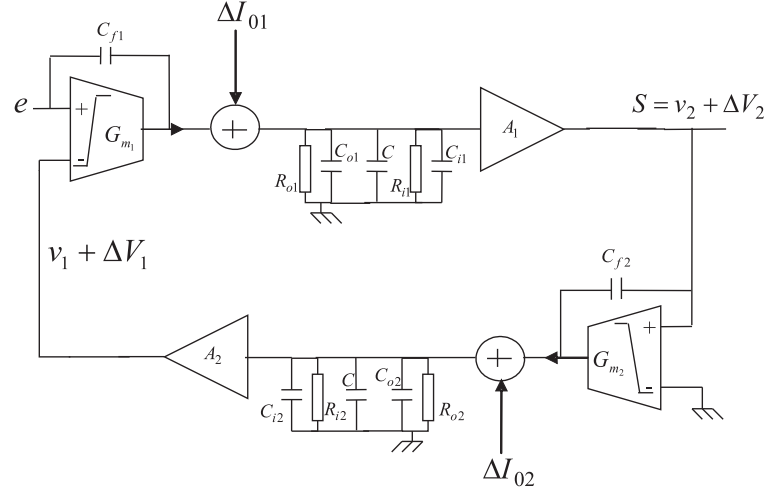

Fig. 4. Resonators model with perturbations

which is the offset at the input of $G_{m 2}$, can be evaluated with the expression (6) when $\omega \rightarrow 0$. The output offset voltage $\Delta V_{1}$ is obtained by the same way, and is the offset voltage at the input of $G_{m 1}$ :

$$
\left\{\begin{array}{l}
\Delta V_{2}=\frac{1}{\alpha}\left(-\frac{A_{1}}{R_{2}} \Delta I_{01}-A_{1} A_{2} G_{m 1} \Delta I_{02}\right) \\
\Delta V_{1}=\frac{1}{\alpha}\left(A_{1} A_{2} G_{m 2} \Delta I_{01}-\frac{A_{2}}{R_{1}} \Delta I_{02}\right)
\end{array}\right.
$$

where $\alpha=\frac{1}{R_{1} R_{2}}+G_{m 1} G_{m 2} A_{1} A_{2} . \Delta V_{2}$ and $\Delta V_{1}$ are the offsets obtained if $G_{m 1}$ and $G_{m 2}$ are both tuned. But, only one transconductance is often tuned, so only the contribution of $\Delta I_{01}$ or of $\Delta I_{02}$ has to be considered. For each of these configurations, the maximal allowed value for $\Delta I_{01}$ or $\Delta I_{02}$ for a correct functioning range will be defined. A correct functioning range will be in our case when none of the transconductance amplifiers enters its saturation zone, with an input signal at resonance. The gain at resonance is $G_{\max }, e$ is the input signal and $V_{\text {sat }}$ is the maximal voltage range at the input of transconductance amplifiers for not being in saturation zone. For the transconductances $G_{m 1}$ and $G_{m 2}$ not to saturate, the following expressions must be verified:

$$
\left\{\begin{array}{l}
\left|\Delta V_{2}\right|<\left(V_{\text {sat }}-G_{\max } e\right) \\
\left|\Delta V_{1}\right|<\left(2 V_{\text {sat }}-e\left(G_{\max } G_{m 2} A_{2} R_{2}+1\right)\right)
\end{array}\right.
$$

Resulting in maximal allowed offsets for $G_{m 2}$ not to saturate:

$$
\left\{\begin{aligned}
\left|\Delta I_{02}\right|_{G_{m 2}} & <\frac{1}{A_{1} A_{2} G_{m 1}} \alpha\left(V_{\text {sat }}-G_{\max } e\right) \\
\left|\Delta I_{01}\right|_{G_{m 2}} & <\frac{R_{2}}{A_{1}} \alpha\left(V_{\text {sat }}-G_{\max } e\right)
\end{aligned}\right.
$$

and maximal allowed offsets for $G_{m 1}$ not to saturate:

$$
\left\{\begin{aligned}
\left|\Delta I_{02}\right|_{G_{m 1}} & <\frac{R_{1}}{A_{2}} \alpha\left(2 V_{\text {sat }}-e\left(G_{\max } G_{m 2} A_{2} R_{2}+1\right)\right) \\
\left|\Delta I_{01}\right|_{G_{m 1}} & <\frac{1}{A_{1} A_{2} G_{m 2}} \alpha\left(2 V_{\text {sat }}-e\left(G_{\max } G_{m 2} A_{2} R_{2}+1\right)\right)
\end{aligned}\right.
$$


Considering that $R_{2} / A_{1}$ and $R_{1} / A_{2}$ are usually several times larger than $1 / A_{1} A_{2} G_{m 2}$ and $1 / A_{1} A_{2} G_{m 1}$, these are $\left|\Delta I_{01}\right|_{G_{m 1}}$ and $\left|\Delta I_{02}\right|_{G_{m 2}}$ which are imposing the maximal allowed offsets. For example, the maximal allowed offsets values are calculated for $V_{\text {sat }}= \pm 200 \mathrm{mV}, e=5 \mathrm{mV}, R_{1}=R_{2}=500$ $\Omega, G_{m 1}=G_{m 2}=7.5 \mathrm{mS}, G_{\max }=13$. The obtained values are: $\left|\Delta I_{01}\right|_{G_{m 1}}=1.2 \mathrm{~mA},\left|\Delta I_{02}\right|_{G_{m 1}}=4.6 \mathrm{~mA},\left|\Delta I_{01}\right|_{G_{m 2}}$ $=4.1 \mathrm{~mA}$ and $\left|\Delta I_{02}\right|_{G_{m 2}}=1.1 \mathrm{~mA}$. So the maximal allowed offsets are $\Delta I_{02}=1.1 \mathrm{~mA}$ and $\Delta I_{01}=1.2 \mathrm{~mA}$.

\section{EXAMPLE}

The purpose is to evaluate the central frequency tuning range of the circuit presented in fig. 5 using the tolerance to current offset. The circuit has been designed with Pspice using the design kit of the GaAs P-HEMT $0.2 \mu m$ technology. It is supplied by $\pm 5 \mathrm{~V}$. The integrated capacitors $C$ are chosen the highest as possible $(1 \mathrm{pF})$ for robustness considerations. The circuit contains external feedback capacitors $\left(C_{f 1}=C_{f 1}=\right.$ $0.3 p F$ ) to increase $Q$ and this make possible to take weak load resistances $(\mathrm{R}=500 \Omega)$, in order to simplify the realization. The value of the product $G_{m 1} G_{m 1}$ has been chosen in order to

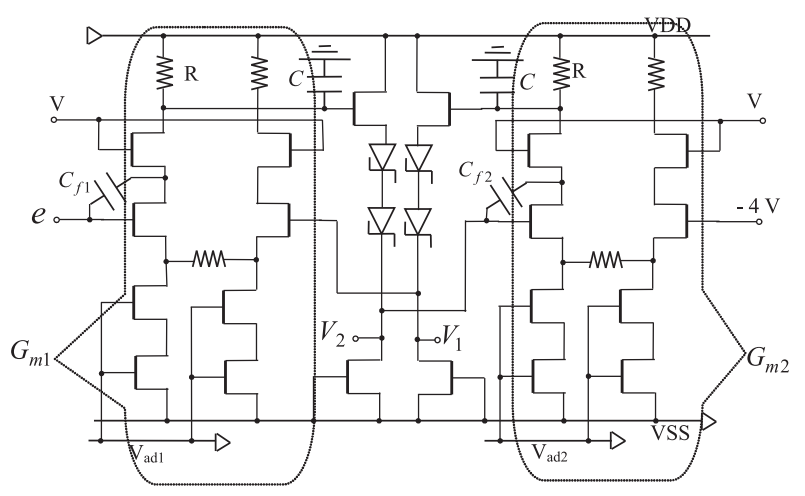

Fig. 5. GaAs P-HEMT $0.2 \mu m$ Gm-C resonator

obtain, a central frequency of about $790 \mathrm{MHz}$. For this case, $G_{m 1}$ and $G_{m 2}$ are chosen equal to simplify the realization (7.5 mS). It results in a quality factor of about 10 . As seen in section II., it is possible to increase it by increasing $G_{m 1}$ and decreasing $G_{m 2}$, but it is not the purpose here. Table I shows the performances obtained for the adjustment of $V_{a d 2}$ on the bias current of $G_{m 2}$ and the adjustment of $V_{a d 1}$ on the bias current of $G_{m 1}$. The current offset tolerance defined in section III is not totally used in order to keep all the transistors in the saturate functioning zone. The figure 6 shows the frequency response of the resonator in these two cases. Varying $G_{m 1}$ and $G_{m 2}$ results in the same variation of the central frequency from about $750 \mathrm{MHz}$ to $810 \mathrm{MHz}$. However, varying $G_{m 1}$ leads to more important variations of the quality factor as discussed in section III A).

\section{CONClusion}

This paper has discussed the ability of tuning $\mathrm{Gm}-\mathrm{C} \mathrm{GaAs}$ resonators. Some general design methodological elements as well as preferential ways of tuning, valid whatever the
TABLE I

Performances of THE TUNABle Resonator

\begin{tabular}{|c|c|c|c|}
\hline$V_{a d 2}$ & $-4.92 \mathrm{~V}$ & $-5 \mathrm{~V}$ & $-5.1 \mathrm{~V}$ \\
\hline$I_{02}+\Delta I_{02}$ & $(2.8+0.6) \mathrm{mA}$ & $2.8 \mathrm{~mA}$ & $(2.8-0.8) \mathrm{mA}$ \\
\hline central frequency & $809 \mathrm{MHz}$ & $796 \mathrm{MHz}$ & $753 \mathrm{MHz}$ \\
quality factor & 10.8 & 10.3 & 7.33 \\
$G_{\max }$ & 10.4 & 9.9 & 7.6 \\
\hline \hline$V_{a d 1}$ & $-4.92 \mathrm{~V}$ & $-5 \mathrm{~V}$ & $-5.1 \mathrm{~V}$ \\
\hline$I_{01}+\Delta I_{01}$ & $(2.8+0.6) \mathrm{mA}$ & $2.8 \mathrm{~mA}$ & $(2.8-0.8) \mathrm{mA}$ \\
\hline central frequency & $809 \mathrm{MHz}$ & $796 \mathrm{MHz}$ & $751 \mathrm{MHz}$ \\
quality factor & 14 & 10.3 & 5.41 \\
$G_{\max }$ & 14 & 9.9 & 5.11 \\
\hline
\end{tabular}
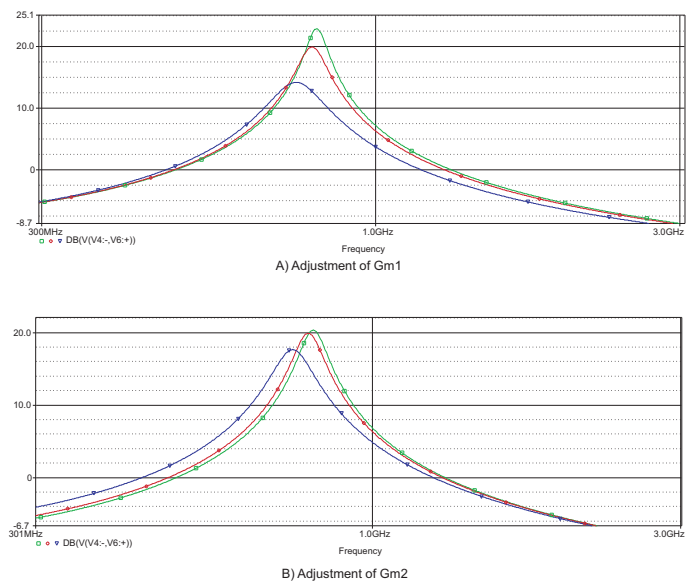

Fig. 6. Adjustment of central frequency. A) by $G_{m 1}$, B) by $G_{m 2}$

technology, have been presented. It is shown that adding an external feedback capacitor on the transconductance amplifiers can help to obtain the desired quality factor. The tolerance of a typical $\mathrm{Gm}-\mathrm{C}$ GaAs structure to current offsets, introduced by the tuning of the central frequency, is determined. Using this tolerance a GaAs P-HEMT simulated circuit demonstrates a tuning central frequency range of $750 \mathrm{MHz}$ to $810 \mathrm{MHz}$.

\section{REFERENCES}

[1] Moezzi, M. and Zanbaghi, R. and Atarodi, M. and Tajalli, A.A QEnhanced Biquadratic Gm-C Filter for High Frequency Applications, 13th IEEE International Conference on Electronics, Circuits and Systems (ICECS'06), pp 248-251, 2006.

[2] Gao, Z.Q. and Yu, M.Y. and Lai, F.C. and Ye, Y.Z. A High $Q \mathrm{Gm}-\mathrm{C}$ Filter for Multi-band Wireless Applications, 8th International Conference on Solid-State and Integrated Circuit Technology, pp 1733-1735, 2006.

[3] Xiao, H. and Schaumann, R. A 5.4-GHz high-Q tunable active-inductor bandpass filter in standard digital CMOS technology, Analog Integrated Circuits and Signal Processing (Springer), vol. 51, nb. 1, pp 1-9, 2007.

[4] Bakken, T. and Choma, J. Gyrator-Based Synthesis of Active On-Chip Inductances, Analog Integrated Circuits and Signal Processing (Springer), vol. 34, nb. 3, pp 171-181, 2003.

[5] Haigh, DG and Toumazou, C. High frequency GaAs transconductors and other building blocks forcommunication, IEEE International Symposium on Circuits and Systems, pp 1731-1735, 1990.

[6] Wu, P. and Schaumann, R. Design of a 1-GHz self-resonance integrated GaAs gyrator-C inductor, 33rd Midwest Symposium on Circuits and Systems, pp 362-365, 1990. 